\title{
New insights into Brunner syndrome and potential for targeted therapy
}

\begin{abstract}
Palmer E.E., Leffler M., Rogers C., Shaw M., Carroll R., Earl J., Cheung N.W., Champion B., Hu H., Haas S.A., Kalscheuer V.M., Gecz J., Field M. New insights into Brunner syndrome and potential for targeted therapy. Clin Genet 2016: 89: 120-127. (C) John Wiley \& Sons A/S. Published by John Wiley \& Sons Ltd, 2015

We report two families with Brunner syndrome living in one state of Australia. The first family had a predicted protein-truncating variant of monoamine oxidase A (MAOA) (p.S251KfsX2). Affected males had mild intellectual disability (ID), obsessive behaviour, limited friendships and were introverted and placid during clinical interview. The family disclosed episodic explosive aggression after a diagnosis was made. The second family had a missense variant in MAOA (p.R45W). Affected males had borderline-mild ID, attention deficit disorder and limited friendships. One had a history of explosive aggression in childhood and episodic symptoms of flushing, headaches and diarrhoea. Their carrier mother had normal intelligence but similar episodic symptoms. Characteristic biochemical abnormalities included high serum serotonin and urinary metanephrines and low urinary 5-hydroxyindoleacetic acid (5-HIAA) and vanillylmandelic acid (VMA). Symptomatic individuals in the second family had particularly high serotonin levels, and treatment with a serotonin reuptake inhibitor and dietary modification resulted in reversal of biochemical abnormalities, reduction of 'serotonergic' symptoms and behavioural improvement. Brunner syndrome should be considered as a cause of mild ID with paroxysmal behavioural symptoms. It can be screened for with serum/urine metanephrine and serotonin measurement. Cautious treatment with a serotonin reuptake inhibitor, dietary modifications and avoidance of medications contraindicated in patients on monoamine oxidase inhibitors can improve symptoms.
\end{abstract}

\section{Conflict of interest}

All authors declare no conflict of interest.

\author{
E.E. Palmer ${ }^{a, b}$, M. Leffler ${ }^{a}$, \\ C. Rogers ${ }^{a}$, M. Shaw ${ }^{c}$, \\ R. Carrollc, J. Earl ${ }^{\mathrm{d}, \mathrm{e}}$, \\ N.W. Cheung d,f , B. Champion ${ }^{\mathrm{d}, \mathrm{f}}$, \\ H. Hug , S.A. Haas', \\ V.M. Kalscheuerg, J. Gecz ${ }^{\mathrm{c}}$ \\ and M. Field ${ }^{\mathrm{a}}$
}

aDepartment of Clinical Genetics, GOLD (Genetics of Learning Disability) service, Waratah, New South Wales, Australia, bUniversity of New South Wales, Waratah, New South Wales, Australia, 'School of Paediatrics and Reproductive Health and Robinson Institute, The University of Adelaide, Adelaide, South Australia, Australia , dDepartment of Paediatrics and Child Health, University of Sydney, Sydney, New South Wales, Australia, 'Department of Biochemistry, The Children's Hospital at Westmead, Sydney, New South Wales, Australia, 'Department of Endocrinology, Nepean Hospital, Sydney, New South Wales, Australia, 9Department of Human Molecular Genetics , and hepartment of Computational Molecular Biology, Max Planck Institute for Molecular Genetics, Berlin, Germany

Key words: autism spectrum disorder Brunner syndrome - diagnosis intellectual disability - MAO-A deficiency - serotonin - treatment

Corresponding author: Michael Field, Clinical Geneticist, Genetics of Learning Disability Service, Hunter Genetics, Cnr Turton and Tinonee Roads, Waratah, New South Wales 2298, Australia. Tel: +61 (0)2 49853100; fax: +61 (0)2 4985 3105; e-mail: Mike.Field@health.nsw.gov.au

Received 29 January 2015, revised and accepted for publication 18 March 2015 
Brunner syndrome (OMIM 300615) was first described in 1993 in a large Dutch kindred. Linkage to Xp11-21 was obtained, and biochemical evidence of monoamine oxidase A (MAOA) deficiency and a subsequent protein-truncating variant in the MAOA gene was identified $(1,2)$. The affected males had non-syndromic borderline-mild intellectual disability (ID) with stress-induced aggressive outbursts. Aberrant sexual behaviour and episodic night terrors were characteristic. Subsequently, there has been debate regarding possible influences of polymorphisms in the MAOA gene and behavioural dysinhibition, especially in the context of childhood stress exposure $(3,4)$. Brunner syndrome because of pathogenic variants in the MAOA gene has been infrequently observed despite active screening of $\mathrm{X}$-chromosome-linked intellectual disability (XLID) cohorts (5). We are aware of two further reports: one family, genetically characterised herein, and previously only identified biochemically (6) and another recent case report (7) of males with variable ID/autism spectrum disorder, aggressive behaviour, a missense variant in $M A O A$ and abnormal urinary monoamines.

There are two genes coding for two specific isoforms of monoamine oxidase (MAO), $M A O A$ and $M A O B$, located at Xp11.2. The MAO enzymes have key roles in oxidative deamination of the neurotransmitters serotonin, dopamine, adrenaline and noradrenaline, as well as metabolising minor amines including tyramine. MAOA is more biologically active and loss of function of MAOB alone because of a partial gene deletion has not been associated with biochemical or clinical sequelae (8).

\section{Materials and methods}

\section{Clinical description of the families}

Family $H$

The pedigree (Fig. 1) consisted of two non-dysmorphic well-grown brothers III:1, 59 years, and III:4, 52 years, with mild ID and essential tremor (clinical details Table 1). They had an affected maternal uncle (II:3) who died at 60 years of age following a motor vehicle accident (unavailable for genetic testing). Notably, behaviour problems were not reported on initial assessment with both males appearing very passive during clinical interview. Direct questioning later revealed III:1's history of impulsivity, school expulsions and violent episodes involving property damage and aggression towards family members. III:4 had a history of explosive temper, affecting his ability to maintain employment, as well as limited interests and obsessive behaviour with significant hoarding. III:4 would occasionally become infatuated with female acquaintances. Both males had difficulties with sleep onset but no history of night terrors. Neither affected male had stereotypical hand movements although III:4, repetitively, combs his hair and both have infrequent involuntary body twitches and essential tremor. No anti-depressant or psychotropic medication had been prescribed. No specific serotonergic symptoms or worsening of behaviour with high tyramine foods or medication were noted.
Family $R$

This family was previously reported by Cheung and Earl (6). An adult female patient of normal intelligence presented with paroxysmal episodes of flushing, diarrhoea, headache and palpitations. She was initially suspected to have carcinoid syndrome, but urinary 5-hydroxyindoleacetic acid levels, abdominal and chest computerized tomography (CT) scans, and somatostatin receptor scan were normal. Further investigation identified raised serum and platelet serotonin and low levels of serotonin metabolites compatible with MAOA deficiency. We recently reviewed this family (Table 1). III:4 has two adult sons aged 33 (IV:5) and 31 (IV:6) years, both of whom have borderline-mild ID and attention-deficit hyperactivity disorder (ADHD). IV:5 had a history of impulsive behaviour and aggressive outbursts in childhood initially treated as epileptic without any noticeable benefit from medication. From adolescence, IV:5 had symptoms of episodic flushing, diarrhoea and headache. IV:6 had difficulties sustaining friendships and obsessive symptoms. IV:5 had recurrent severe nightmares from childhood into adolescence. Neither affected male had persistent abnormal sexual behaviour although neither have had a sustained relationship with a female partner. Neither affected male have stereotypical hand movements; both have an essential tremor (as does their unaffected father), and IV:5 has occasional finger and leg 'twitches'.

All affected individuals in Family $\mathrm{R}$ notice an exacerbation of serotonergic symptoms when they consume food and drink high in tyramine, especially beer, cheese and 'vegemite' (yeast extract). These foods were a trigger for the aggressive outbursts for IV:5. Patients III:4, IV:5 and IV:6 were cautiously commenced on $50 \mathrm{mg} /$ day of sertraline, a selective serotonin reuptake inhibitor (SSRI). The dose of medication was very slowly increased under hospital admission supervision. There were initial exacerbation of serotonergic symptoms, but then III:4 and IV:5 (the more severely affected individuals) reported reduction in the frequency of headaches and flushing, severity of diarrhoea and improvement in mood. A reduction in aggressive outbursts for IV:5 was noted by his family. III:4 reported similar beneficial effects on venlafaxine ( $37.5 \mathrm{mg} /$ day increased to $75 \mathrm{mg} /$ day). Complete normalisation of biochemistry was demonstrated on treatment with sertraline (6); however, even on treatment, affected individuals report re-emergence of milder serotonergic symptoms with dietary indiscretions.

III:5 was recently briefly trialled off sertraline 10 years after treatment was commenced, on request of his family. Repeat bioamine profiling (on and off SSRI medication) demonstrated a normalisation of serum serotonin and normetanephrine/metanephrine on medication and re-elevation off medication (Fig. 2 and Table S1, Supporting Information). Off medication, he had frequent episodes where he was flushed, diaphoretic, disorientated, tachycardic and mildly hypertensive, and often unable to work. 
Palmer et al.

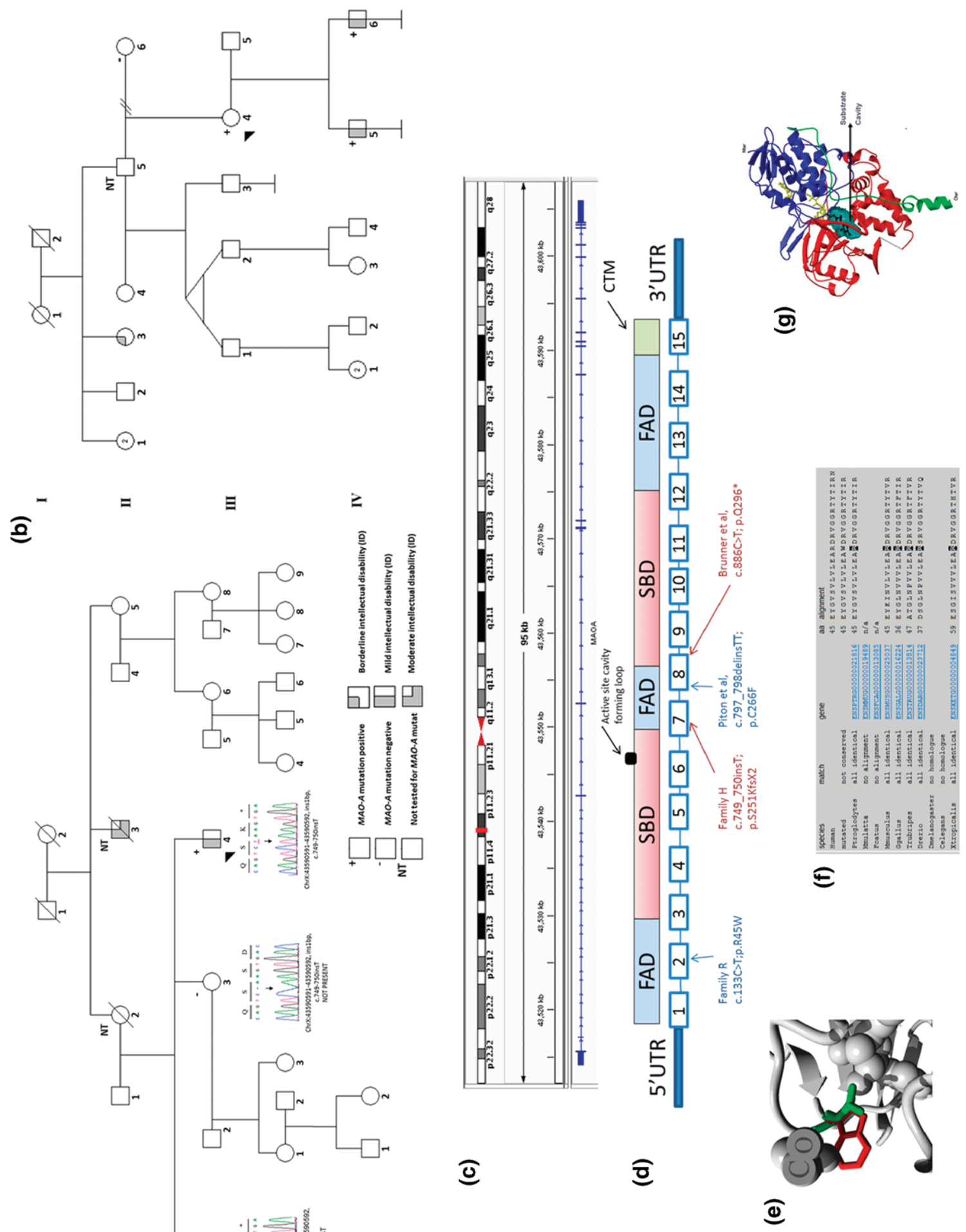

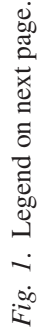




\section{New insights into Brunner syndrome}

\section{X-exome sequencing}

Family $\mathrm{H}$

The proband from this family, III:4, was investigated by $\mathrm{X}$-chromosome exome capture as described in $\mathrm{Hu}$ et al. (9).

\section{Sanger sequencing}

\section{Family $\mathrm{H}$}

DNA extracted from whole-blood (QIAamp DNA blood maxi kit; Qiagen, Limburg, The Netherlands) was part of a large X-chromosome exome sequencing study (9). Only one affected individual was sequenced (Illumina GAIIx; Max Planck Institute for Molecular Genetics, Berlin, Germany). Confirmation of variants by Sanger sequencing was carried out using standard methods.

\section{Family $R$}

DNA was extracted from lymphocytes, and DNA Sanger sequencing of the MAOA gene (NM_000240.2) was performed using standard methodology (Laboratorium Genoomdiagnostiek, UMS St Radboud, Nijmegen, The Netherlands).

\section{Bioamine assays}

Plasma noradrenaline and adrenaline levels, serum serotonin levels, and urine levels of catecholamines, metanephrine, normetanephrine and 5HIAA levels were quantitated by high-performance liquid chromatography (HPLC) with electrochemical detection. Plasma metanephrine and normetanephrine levels were quantitated by liquid chromatography-mass spectrometry (LCMS).

\section{Results}

Family $\mathrm{H}$

A single base pair insertion in exon 5 of the $M A O A$ gene was identified. This DNA variant is predicted to result in a truncated MAOA protein, c.749-750insT; p.S251KfsX2 (using NM_000240) (Fig. 1d).The variant was present in both males, and their mother was assumed to be an obligate carrier.
The deleterious effect of the variant was confirmed by profiling the bioamine levels in blood and urine in this family (Fig. 2 and Table S1). These biochemical results are similar to those previously reported by patients with Brunner syndrome (1, 6, 7; Fig. 2 and Table S1).

\section{Family $\mathrm{R}$}

Sequencing revealed a rare novel missense variant c.133C $>\mathrm{T}$ (p.Arg45Trp) in MAOA (transcript ENSP00000340684) (Fig. 1d). This variant is not present in the ExAC dataset [Exome Aggregation Consortium, Cambridge, MA (http://exac.broadinstitute.org), accessed December 2014], affects an evolutionarily conserved residue (down to Xenopus) (Fig. 1f) and is predicted to have a functional impact based on in silico prediction software including PROVEAN [Protein Variation Effect Analyser (http://provean.jcvi.org/index.php)] (predicted pathogenic - score -6.7) and MutATionTASTER [Mutation Taster (http://mutationtaster.org/)] (disease causing). The variant introduces a larger and more hydrophilic amino acid in the oxidase [flavin adenine dinucleotide (FAD) binding] domain of the protein (Fig. 1g), predicted to impact on substrate binding and protein folding (Fig. 1e). The variant was present in both affected males, and their symptomatic mother; but not present in the maternal grandmother. The maternal grandfather was unavailable for testing. All variants have been submitted to CLINVAR [ClinVar (www.ncbi.nlm.nih.gov/clinvar/].

Bioamine levels for affected family members are summarised in Fig. 2 (detailed information in Table S1). MAOA substrates serotonin and normetanephrine were elevated, and bioamine metabolites were in the low normal range. The effect on serotonin metabolism was more marked than that on catecholamine metabolism.

\section{Discussion}

Brunner syndrome has been an uncommonly diagnosed cause of XLID with only two new cases reported in the last 20 years $(6,7)$. The identification of at least two cases in the Australian state of New South Wales suggests that the scarcity of cases may represent problems with ascertainment, rather than rarity of the phenotype. In both of the families that we report, the degree of developmental delay in affected males was mild and semi-open

Fig. 1. Pedigrees of the two families and characterisation of $M A O A$ variants. (a) Pedigree for Family $\mathrm{H}$ showing Sanger results of $M A O A$ sequencing. Primers flanking $M A O A$ exon 7 were used to amplify and sequence gDNA, F - 5' -TGG CCT GTG ACT TTC TGG A-3' and R - 5'-GCA GGC GTG AAA AAT CAT CT-3'. Arrow indicates nucleotide variant site (Hg19: ChrX: 43590591-43590592, ins1bp, p.S251KfsX2). (b) Pedigree for Family R. (c) Position of MAOA gene on X-chromosome (integrative genomics viewer, IGV); (d) location of functional domains of MAOA protein and position of variants for Family $\mathrm{R}$ and $\mathrm{H}$ and those described by Brunner et al. (2) and Piton et al. (7). FAD (blue boxes) = flavin adenine dinucleotide binding domains: residues 13-88, 220-294, and 400-462; SBD (red boxes) = substrate-binding domain: residues 89-219 and 295-399; CTM (green box) C-terminal membrane region: residues 463-506. The active site cavity-shaping loop (residues 210-216) is depicted as black box. The missense variant for Family R lies within a FAD-binding domain, as does the missense variant for the family described by Piton et al. (7). (e) Close-up of the predicted structural effect of Family R variant (R45W) - the missense variant is predicted to introduce a larger, more hydrophobic residue that will cause an ionic interaction within the protein to be lost. The protein is coloured grey, the side chains of both the wild-type and the mutant residue are shown and coloured green and red, respectively (16); (f) conservation of residue R at position 45 (highly conserved down to Xenopus tropicalis) (15) (g) Reprinted with permission from Edmondson, Binda and Mattevi, 2007. Ribbon diagram of human MAOA protein, colours follow descriptions in b. R arginine, $\mathrm{W}$, tryptophan. 
Palmer et al.

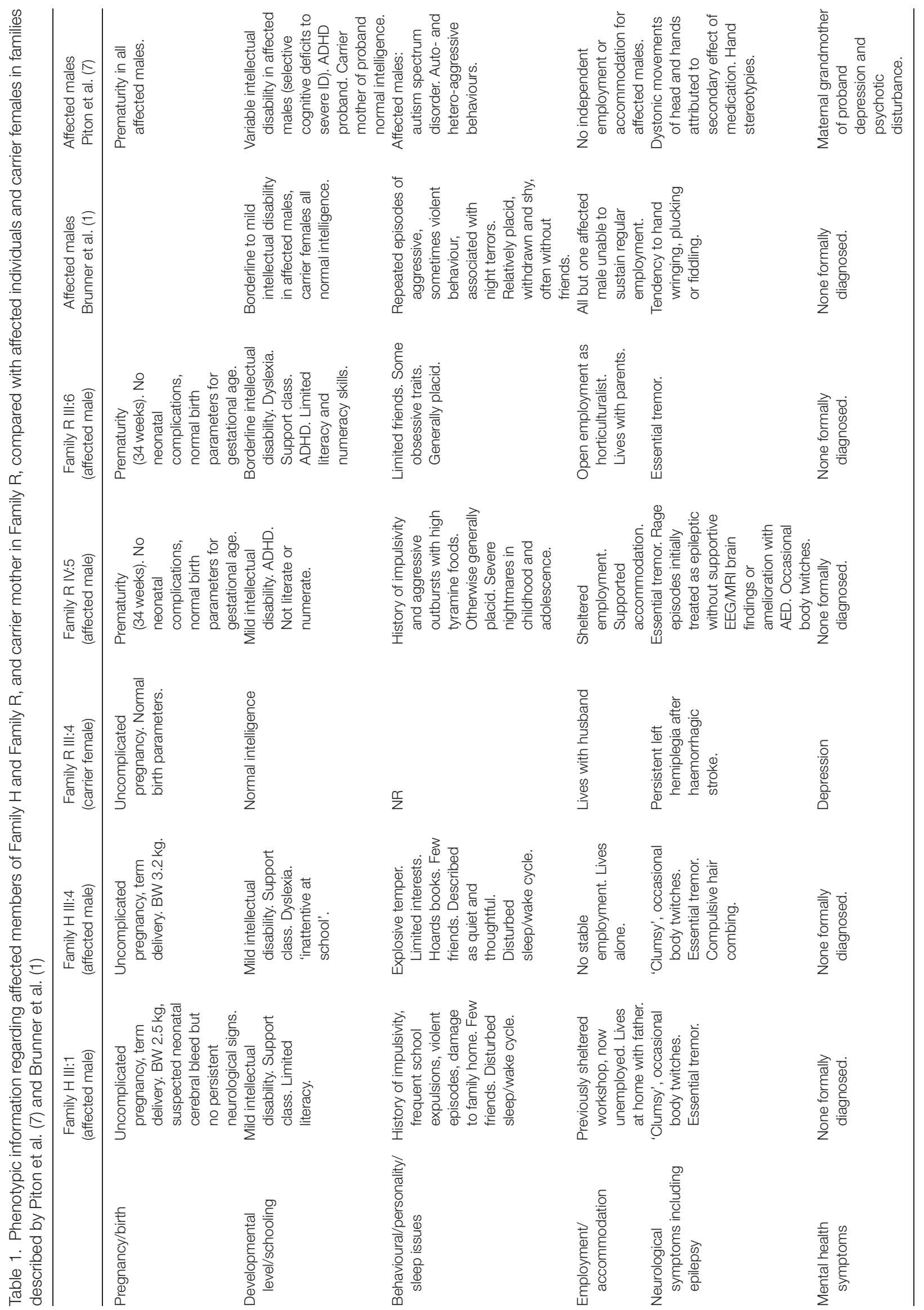


New insights into Brunner syndrome

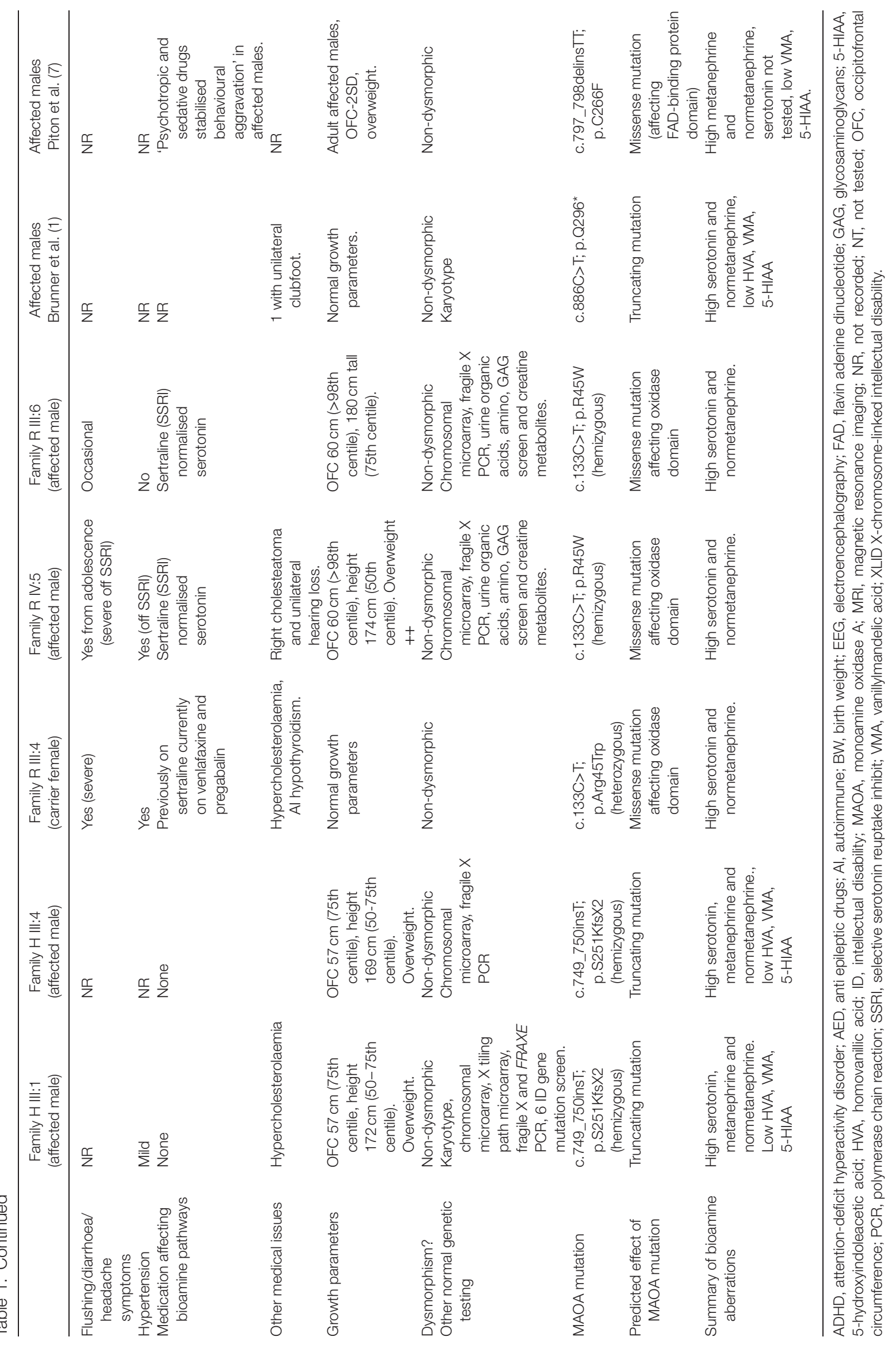



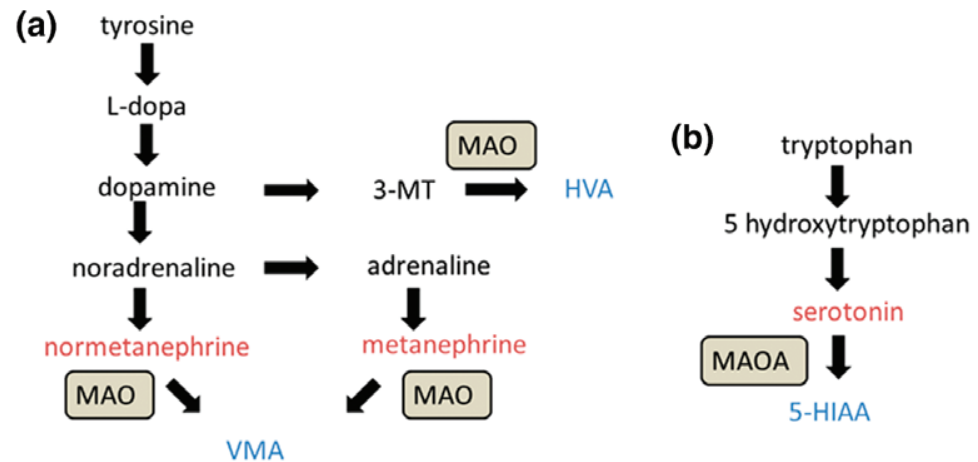

(c)

\begin{tabular}{|c|c|c|c|c|c|}
\hline & Family H & $\begin{array}{c}\text { Family R (off } \\
\text { medication) }\end{array}$ & $\begin{array}{c}\text { Family R (on } \\
\text { medication) }\end{array}$ & $\begin{array}{c}\text { Brunner } \\
\text { family }\end{array}$ & $\begin{array}{c}\text { Piton } \\
\text { family }\end{array}$ \\
\hline dopamine & $\rightarrow$ & $\rightarrow$ & $\rightarrow$ & NT & NT \\
\hline HVA & $\downarrow$ & $\rightarrow$ & NT & $\downarrow$ & $\rightarrow$ \\
\hline serotonin & $\uparrow$ & $\uparrow$ & $\rightarrow$ & $\uparrow$ & $\uparrow$ \\
\hline 5-HIAA & $\downarrow$ & $\rightarrow$ & $\rightarrow$ & $\rightarrow$ & $\downarrow$ \\
\hline normetanephrine & $\uparrow$ & $\uparrow$ & $\rightarrow$ & $\uparrow$ & $\uparrow$ \\
\hline metanephrine & $\uparrow$ & $\uparrow$ & $\rightarrow$ & $\uparrow$ & $\uparrow$ \\
\hline VMA & $\downarrow$ & $\downarrow$ & NT & $\downarrow$ & $\downarrow \rightarrow$ \\
\hline
\end{tabular}

Fig. 2. Changes in bioamine metabolism in patients with monoamine oxidase A (MAOA) deficiency. (a) Serotonin metabolism pathway with changes in metabolites detected in patients affected by MAOA deficiency. MAOA substrate serotonin (red) elevated and breakdown product 5-hydroxyindoleacetic acid (5-HIAA) (blue) decreased or unchanged. (b) Catecholamine metabolism pathway with changes in metabolites detected in patients affected by MAOA deficiency. MAOA substrates normetanephrine and metanephrine (red) elevated and breakdown products vanillylmandelic acid (VMA) and homovanillic acid (HVA) (blue) decreased or unchanged. (c) Comparison of bioamine metabolite results for affected patients in Family H, R, Brunner et al. (1) and Piton et al. (7) families. Elevations in MAOA substrates normetanephrine, metanephrine and serotonin levels are the most consistent biochemical signature for MAOA deficiency. These metabolite levels were completely normalised in affected individuals from Family R treated with a selective serotonin reuptake inhibitor (SSRI) (column 4). Absolute values are listed in Table S1. NT, not tested.

employment, and independent living was possible. It is conceivable that the cohorts of patients that have previously been screened for Brunner syndrome had more significant cognitive disability. There has also been debate and potential misinterpretation of the behavioural characteristics of affected males (10, 11): episodic impulsive behaviour leading at times to physical aggression seems to be the most important behavioural clue. Affected males also had autistic features, including lack of friendships, difficulties interpreting female relationships, narrow interests and obsessional collecting. Similar characteristics were reported in the family described by Piton (7). Parasomnias, including night terrors, and subtle neurological symptoms of tremor, stereotypical hand movements or occasional body twitches also appear to be characteristic. Abnormal bioamines are an effective way to follow up the significance of any detected variants in $M A O A$ (e.g. from genomic screening).

Specific questioning regarding 'serotonergic syndrome' symptoms (episodic flushing, headaches and diarrhoea) and the exacerbation of physical and behavioural symptoms with high tyramine-containing foods and drinks, such as cheese and yeast extract, may also be diagnostically helpful. These symptoms are not mentioned in the case reports of Brunner and Piton, but may not have been specifically elicited. Serotonergic symptoms may not be universal for Brunner syndrome: there may be genotype-phenotype correlation. They were not present in affected members of Family H, who had a loss of function variant, but were prominent in Family R who had a missense variant. The affected members of Family R had particularly high serotonin levels off medication. It is possible that the conformational change of the oxidase-binding site in affected members of Family R may selectively affect serotonin metabolism more than other bioamines (Fig. 2). The MAOA missense variant Y444F has such a specific effect (12). Additional functional work and evaluation of further clinical cases is required to investigate this further.

We suggest that behavioural issues in individuals affected by Brunner syndrome are related to elevated serotonin, although the mechanism is incompletely understood at the synaptic level. Elevated platelet serotonin has been associated with autism spectrum disorder (13) and a low efficiency promoter region polymorphism within the MAOA gene associated with autism severity and aggression (14). An MAOA-deficient mouse model suggests serotonin elevation may result in an increase in the frequency and intensity of physical aggression between male mice (15). 
The amelioration of physical and behavioural symptoms with SSRI/SNRI (seratonin-norepinephrine reuptake inhibitor) medication in Family $\mathrm{R}$ is difficult to explain comprehensively. This appears paradoxical, as the anti-depressant/anti-anxiolytic effect of this medication is postulated to be because of an increase of monoamines at brain synapses, and these medications are contraindicated in patients taking a monoamine oxidase inhibitor due to the risk of precipitating serotonergic syndrome. However, there is supportive animal model data that administration of the SSRI fluoxetine reduces aggressive behaviour and perseverative responses in $M A O A$ knockout mice and improves social deficits and perseverative responses in mice with a hypomorphic MAOA variant (16). The authors were surprised by this treatment effect as they had anticipated that the SSRI would worsen symptoms.

Earl and Cheung (6), who originally recommended a therapeutic trial of SSRI in Family R, postulated that symptoms related to serotonergic excess would be reduced over time because of reduction of peripheral serotonin. Blood serotonin levels fall in individuals treated with SSRI's due to inhibition of platelet serotonin transporters, platelets being the major storage site of serotonin in the blood (17). The exact effect of SSRI/SNRI medication acutely and chronically in different brain regions even in individuals with normal MAOA function is far from understood (18), and it is difficult to say whether improvements in mood and behaviour in treated members of Family $\mathrm{R}$ were purely because of relief of physical symptoms or an additional central effect.

Cautious treatment with an SSRI may be useful in other families with Brunner syndrome, particularly in individuals with high serotonin levels and debilitating 'serotonergic symptoms'. Such treatment trials carry the risk of precipitating life-threatening serotonergic syndrome as patients with Brunner syndrome likely have increased stores of serotonin because of loss of MAOA function. We would recommend that an SSRI/SNRI be started at the lowest possible dose with very slow escalation and close cardiovascular and neurological monitoring over at least a 2-week hospital admission.

The potential of tyramine-containing foods and certain medication (those contraindicated in patients on a monoamine oxidase inhibitor) to precipitate potentially life-threatening serotonergic symptoms points to the additional medical importance of diagnosing Brunner syndrome. We would advise individuals diagnosed with Brunner syndrome to be referred to a dietician, avoid medication contraindicated in patients on monoamine oxidase inhibitors unless under strict medical supervision and be issued with 'Medic-Alert' bracelets.

\section{Supporting Information}

Additional supporting information may be found in the online version of this article at the publisher's web-site.

\section{Acknowledgements}

With many thanks to the family members described in this article and their primary medical teams. The authors thank the Exome Aggregation Consortium and the groups that provided exome variant data for comparison. A full list of contributing groups can be found at http://exac.broadinstitute.org/about. J. G. has been supported by NHMRC Programme Grant 628952 and senior research fellowship 1041920. Part of this work was financed by the EU FP7 project GENCODYS, grant number 241995 (H. H. and V. M. K).

\section{References}

1. Brunner HG, Nelen MR, van Zandvoort $P$ et al. X-linked borderline mental retardation with prominent behavioral disturbance: phenotype, genetic localization, and evidence for disturbed monoamine metabolism. Am J Hum Genet 1993: 52: 1032-1039.

2. Brunner HG, Nelen M, Breakefield XO et al. Abnormal behavior associated with a point mutation in the structural gene for monoamine oxidase A. Science 1993: 262: 578-580.

3. Goldman D, Rosser AA. MAOA-environment interactions: results may vary. Biol Psychiatry 2014: 75: 2-3.

4. Brunner HG. MAOA deficiency and abnormal behaviour: perspectives on an association. Ciba Found Symp 1996: 194: 155-164; discussion $164-157$.

5. Schuback DE, Mulligan EL, Sims KB et al. Screen for MAOA mutations in target human groups. Am J Med Genet 1999: 88: 25-28.

6. Cheung NW, Earl J. Monoamine oxidase deficiency: a cause of flushing and attention-deficit/hyperactivity disorder? Arch Intern Med 2001: 161: 2503-2504

7. Piton $\mathrm{A}$, Poquet $\mathrm{H}$, Redin $\mathrm{C}$ et al. 20 ans après: a second mutation in MAOA identified by targeted high-throughput sequencing in a family with altered behavior and cognition. Eur J Hum Genet 2014: 22: $776-783$.

8. Lenders JW, Eisenhofer G, Abeling NG et al. Specific genetic deficiencies of the A and B isoenzymes of monoamine oxidase are characterized by distinct neurochemical and clinical phenotypes. J Clin Invest 1996: 97: 1010-1019.

9. Hu H, Haas SA, Chelly J et al. X-exome sequencing of 405 unresolved families identifies seven novel intellectual disability genes. Mol Psychiatry 2015. doi: 10.1038/mp.2014.193.

10. Hebebrand J, Klug B. Specification of the phenotype required for men with monoamine oxidase type A deficiency. Hum Genet 1995: 96: 372-376.

11. Brunner H, Ropers H. Reply to Hebebrand and Klug. (Letter). Hum Genet 1995: 96: 374-365.

12. Nandigama RK, Miller JR, Edmondson DE. Loss of serotonin oxidation as a component of the altered substrate specificity in the Y444F mutant of recombinant human liver MAO A. Biochemistry 2001: 40: 14839-14846.

13. Anderson GM. Genetics of childhood disorders: XLV. Autism, part 4: serotonin in autism. J Am Acad Child Adolesc Psychiatry 2002: 41 1513-1516.

14. Cohen IL, Liu X, Lewis ME et al. Autism severity is associated with child and maternal MAOA genotypes. Clin Genet 2011: 79: 355-362.

15. Kulikov AV, Osipova DV, Naumenko VS et al. A pharmacological evidence of positive association between mouse intermale aggression and brain serotonin metabolism. Behav Brain Res 2012: 233: $113-119$.

16. Godar SC, Bortolato M, Castelli MP et al. The aggression and behavioral abnormalities associated with monoamine oxidase A deficiency are rescued by acute inhibition of serotonin reuptake. J Psychiatr Res 2014: 56: $1-9$.

17. Beikmann BS, Tomlinson ID, Rosenthal SJ et al. Serotonin uptake is largely mediated by platelets versus lymphocytes in peripheral blood cells. ACS Chem Neurosci 2013: 4: 161-170.

18. Olivier B. Serotonin: a never-ending story. Eur J Pharmacol 2015: 753: $2-18$. 